\begin{tabular}{|c|c|c|}
\hline Beitr. Ent. & Keltern & ISSN 0005-805X \\
\hline $53(2003) 2$ & S. $437-447$ & 15.12 .2003 \\
\hline
\end{tabular}

\title{
Die Arten der Gattung Oegoconia StAINTON,1854 in Deutschland und ihre Verbreitung in Ostdeutschland
}

\author{
(Lepidoptera: Autostichidae)
}

Mit 40 Figuren

REINHARD SUTTER

\section{Zusammenfassung}

In den ostdeutschen Bundesländern sind 3 Arten der Gattung Oegoconia nachgewiesen. Die Fundorte werden aufgelistet, ebenfalls die Fundorte der untersuchten Exemplare aus den westdeutschen Bundesländern. Von O. novimnundi BUSCK, 1915 liegt aus Baden-Württemberg ein Erstnachweis für Deutschland vor. Die Bestimmungstabelle enthält alle 5 Arten Deutschlands.

\section{Summary}

The genus Oegoconia is represented in the East German states by three species. The records are listed, records from other parts of Germany are added. O. novimundi BUSCK, 1915 is recorded for the first time from Germany (Baden-Württemberg). A key to all five species of Germany is provided.

\section{Vorbemerkungen}

Falter der Gattung Oegoconia STAINTON, 1854 können habituell nicht sicher determiniert werden. HUEMER (1998) hat die Identität der bisher beschriebenen Arten geklärt und ermöglicht die sichere Determination anhand von Genitaluntersuchungen. Zum Zeitpunkt dieser Publikation waren die Vorarbeiten für das Verzeichnis der Schmetterlinge Deutschlands (GAEDIKE \& HEINICKE, 1999) fast abgeschlossen, so dass das Verzeichnis bezüglich der Gattung Oegoconia unvollständig ist und unter O. quadripuncta HAWORTH,1828 falsche Angaben enthält. Zwischenzeitlich hat BIESENBAUM (2002) die rheinisch-westfälischen Funde überarbeitet. Vorliegende Arbeit widmet sich der Verbreitung von Oegoconia in Ostdeutschland. In diesem Zusammenhang wurden aber auch Falter aus den westdeutschen Bundesländern untersucht, deren Fundorte im faunistischen Verzeichnis beigefügt sind. Von O. novimundi BuSCK,1915 ergab sich ein Erstnachweis für Deutschland. Damit sind aus Deutschland 5 Arten nachgewiesen. 
In Deutschland nicht zu erwarten sind O. deluccai AMSEL, 1952 (von Malta, Sizilien, Italien, Kroatien, Pyrenäen), O. ariadne GOZMÁNY,1988 (von Kreta, Karpathos, Samos) und parodia GOZMÁNY, 1988 (von Südspanien, Marokko).

Beigefügte Bestimmungstabelle basiert aus dem oben genannten Grund auf Genitalunterschieden, die Abbildung von Faltern erübrigt sich. Zwecks Übersichtlichkeit der Legenden werden die Funddaten der für die Abbildungen benutzten Tiere in einem Abbildungsverzeichnis gesondert nachgewiesen.

\section{Revidiertes Material}

Die untersuchten Falter für vorliegende Arbeit stammen aus den Sammlungen der nachfolgend genannten Museen, Institute und aus Privatsammlungen:

$\begin{array}{ll}\text { DEI } & \text { Deutsches Entomologisches Institut im ZALF, Eberswalde } \\ \text { MNGE } & \text { Museum für Naturkunde Gera } \\ \text { MNGO } & \text { Museum der Natur Gotha } \\ \text { MNVD } & \text { Museum für Naturkunde und Vorgeschichte Dessau } \\ \text { MTD } & \text { Staatliches Museum für Tierkunde Dresden } \\ \text { NML } & \text { Naturkundemuseum Leipzig } \\ \text { ZNHU } & \text { Museum für Naturkunde der Humboldt-Universität Berlin } \\ \text { ZSM } & \text { Zoologische Staatssammlung München }\end{array}$

Privatsammlungen:

Klaus-RÚdiger Beck (Demitz-Thumitz), Uwe Deutschmann (Rubow), FrIedmar Graf (Großdubrau), Timm Karisch (Dessau), Christoph KAyser (Wietzendorf), Jörg Kellner (Dessau), JOOP H. KUCHLENN (Wageningen/Niederlande), HANS LEUTSCH (Niederoderwitz), JAN LIŠKKA (Praha), Hans-JoAchim Van LOH (Leer), Herbert Pröse (Hof/Saale), Dr. Twan RutTen (Ballenstedt), Dr. Andreas Segerer (München), JaN Skyva (Praha), Dr. Helnut Steuer (Bad Blankenburg), ReinHARD SUTTER (Bitterfeld), SvEN W'AUER (Ebersbach Krs. Löbau) und WOLFGANG WITTLAND (Mönchengladbach).

Allen genannten Herren und Museen sei für die freundliche Ausleihe herzlich gedankt.

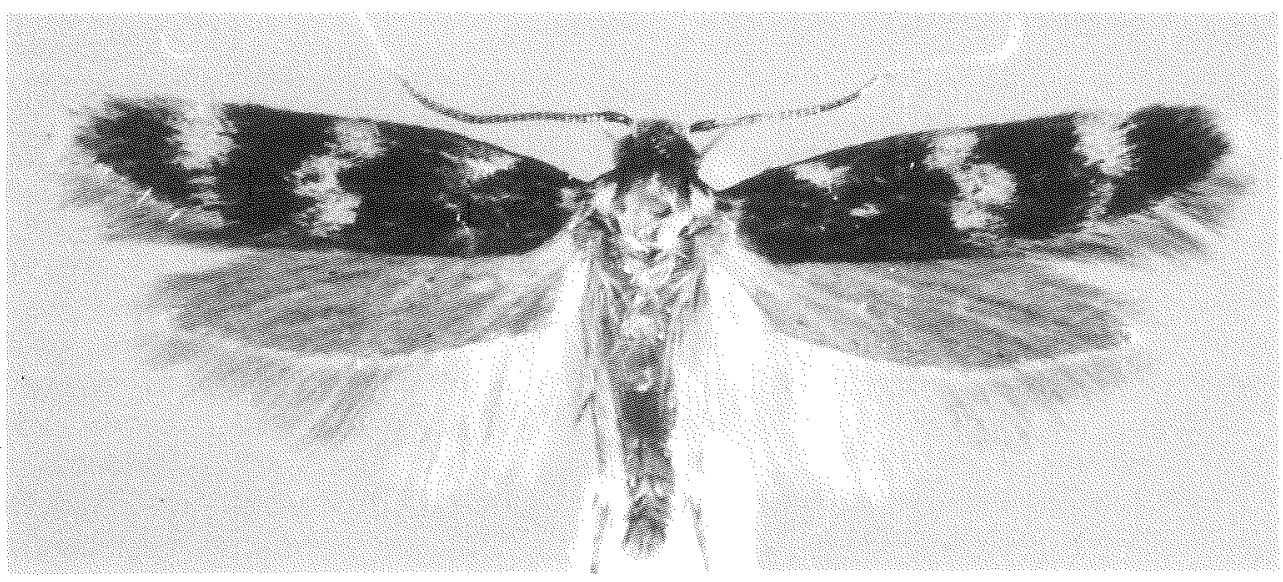

Fig. A: Oegoconia sp. 


\section{Bestimmungstabelle}

우….

Anmerkung: Der Ductus bursae ist vor dem Ostium unterschiedlich stark s-förmig gebogen. Beim Einlegen entsteht dann eine Querfalte, ähnlich Fig. 17. Zur Demonstration der Ductuslängen und der Einschnürung des Ductus vor dem Ostium wurde bei den übrigen Arten der Ductus geglättet eingebettet.

2 Aedoeagus mit auffälligem Cornutibüschel (Fig. 6-7). Abdominalsternit I-II im Körperinneren mit Coremataorgan, einem häutigen Sack mit zahlreichen Duft(?)Haaren (wie Fig. 1) deauratella

- $\quad$ Aedoeagus ohne dieses Cornutibüschel (Fig. 8-15) 3

3 Abdominalsternit I-II mit Coremataorgan (wie Fig. 1) 4

Coremataorgan nicht ausgebildet, nur ein Ansatz sichtbar (Fig. 2-3) 5

4 Aedoeagus mit langem Sklerit in der Vesica (Fig. 8-9) quadripuncta Aedoeagus mit kurzem Sklerit (Fig. 10-11) caradjai

5 Aedoeagus vor der Spitze meist deutlich asymmetrisch (Fig. 12-13), das Sklerit in der Vesica ist länger als bei novimundi. Der Saccus ist je nach Verebnung durchschnittlich breiter (Fig. 4). Bei mitteleuropäischen Exemplaren sind die sklerotisierten Flächen medial längs der Rippen des Sternit I-II einander bogenförmig genähert (Fig. 2) oder miteinander verbunden uralskella Aedoeagus vor der Spitze meist lang und spitz ausgezogen (Fig. 15), selten auch langgezogen und breiter (Fig. 14). Das mehr oder weniger undeutliche Sklerit in der Vesica ist kürzer als bei uralskella. Der Saccus (Fig. 5) ist spitzer. Die sklerotisierten Flächen medial längs der Rippen des Sternit I-I I sind einander nicht genähert (Fig. 3) novimundi

6 Ductus bursae breit und relativ kurz, sklerotisiert, gekörnt (Fig. 16). Signumbasis langoval, am Ende mit größerem Dorn (Fig. 24-26). An den Längsseiten der Basis mehr oder weniger halbrunde, abstehende Flächen deauratella Ductus bursae schmaler (Fig. 17-21). Die variablen Signa von anderem Aufbau (Fig. 22-23, 27-29, 31-34, 36-39) .7

7 Ductus bursae sehr lang, homogen, faltig (Fig. 17). Signumbasis schmal (Fig. 22-23) quadripuncta

Ductus bursae kürzer (Fig. 18-21) 8

8 Ductus bursae im Mittelteil linksseitig häutig und rechtsseitig etwas verstärkt. Ein- 
gelegt zeigt sich linksseitig ein faltiger und rechtsseitig ein glatter Rand (Fig. 18, vgl. auch caradjai Punkt 9). Lappen distal vom Ostium schwach sklerotisiert (Fig. 30). Die Signumbasis schmal (Fig. 27-28). Signum auch anormal mit verbreitertem Dorn und zahlreichen Nebendornen (Fig. 29) .................................novimundi

Signumbasis breit (Fig. 31-34, 36-39) …………………................................... 9

9 Signumbasis (Fig. 31-33) seitlich spitzer auslaufend als bei uralskella. Signumbasis auch mit anormal verbreitertem Dorn (Fig. 34). Ductus bursae mit gekörnter, schwach sklerotisierter und unscharf begrenzter, länglicher Fläche (Fig. 20). Beim Einbetten kann diese Fläche längsgefaltet werden und es entsteht ein mehr oder weniger scharfer Rand (Fig. 19), ähnlich novimundi (Fig. 18). Ductus vor dem Ostium stärker eingeschnürt als bei uralskella. Distal vom Ostium häutig und nur undeutlich lappenförmig (Fig. 35) caradjai Signumbasis seitlich verrundet (Fig. 36-37), selten auch weniger deutlich (Fig. 38). Signum auch mit anormal verbreitertem und stark gekrümmtem Dorn (Fig. 39). Ductus vor dem Ostium schwächer eingeschnürt (Fig. 21). Distal vom Ostium mit schwach sklerotisierten Lappen (Fig. 40) uralskella

\section{Faunistisches Verzeichnis}

Die Angaben zur Flugzeit gelten für Deutschland. Gesicherte Angaben zur Biologie liegen nicht vor. Für die Verbreitung werden nachfolgend nur Angaben von HUEMER (1998) und eigene Ergebnisse verwendet. Für die einzelnen Bundesländer werden die Kürzel vom Verzeichnis der Schmetterlinge Deutschlands (GAEDIKE \& HEINICKE, 1999) übernommen.

\section{Oegoconia quadripuncta (HAWORTH, 1828)}

Lepidoptera Brittanica 4:557 (Recumaria)

(= deauratella STAINTON, 1849)

Flugzeit: Mitte Juli bis Mitte August $(n=5)$

Verbreitung: England, Frankreich, Deutschland, Spanien.

\section{Nachweise aus Deutschland:}

RP: Neustadt/Pfalz, Elmstein und Dürkheim (ROESLER, coll. ZSM)

BW: Baden-Baden (LINACK, coll. OSTHELDER coll.ZSM)

Anmerkung: Die Angaben bei GAEDIKE \& HEINICKE (1999) für NW, HE, BY und SL sind ungesichert, die für $\mathrm{SN}$ und $\mathrm{TH}$ sind zu streichen. 


\section{Oegoconia novimundi (BUSCK, 1915)}

Proc. ent. Soc. Wash. 17: 84 (Symmoca)

Flugzeit: Ende Juni bis Mitte August ( $\mathrm{n}=8$ )

Verbreitung: Frankreich, Deutschland, Österreich, Spanien, Italien, Kroatien, Serbien, Bulgarien, Mazedonien, Griechenland, Kaukasus, auch Azoren und USA.

Nachweise aus Deutschland:

BW: Heidelberg-Kirchheim (RUTTEN)

\section{Oegoconia caradjai POPEsCU-GorJ \& CAPUŞE, 1965}

Revue Roumaine de Biologie, Ser. Zoologie 10: 6

Flugzeit: Mitte Mai bis Ende August

Verbreitung: Mediterran, nördlich bis England, Niederlande, Deutschland, Tschechien, Slowakei und Balkan, Nachweise auch aus der Türkei, vom Iran, Kaukasus, aus Usbekistan, Tadschikistan, in Nordafrika von Marokko, Algerien, Ägypten, auch aus Neuseeland bekannt.

Nachweise aus Ostdeutschland:

ST: Halle/Saale (O. MÜLLER, coll. ZMHU), Saalberge bei Naumburg (BAUER, coll. ZSM)

Untersuchtes Material aus den übrigen Bundesländern:

NW: Prummern bei Heinsberg (STEEGERS, coll. LEUTSCH), Erkelenz-Borschemich und Mönchengladbach-Rheydt (WITTLAND)

RP: Neustadt/Pfalz (RoESLER, coll. ZSM), Speyer (DISQUE, coll. ZSM), Schloßbökelheim (KIEIN, coll. DEI)

BW: Rheinfelden (O. MÜLLER, coll. ZMHU), Heidelberg (RUTTEN)

\section{Oegoconia uralskella POPESCU-GORJ \& CAPUŞE, 1965}

Revue Roumaine de Biologie, Ser. Zoologie 10: 6

Flugzeit: Ende Juni bis Ende August

Verbreitung: Frankreich, Schweiz, Deutschland, Tschechien, Österreich, Italien, Russland, Kaukasus, Altai. 


\section{Nachweise aus Ostdeutschland:}

SN: Jocketa bei Plauen (HEINICKE, coll. MNGE), Meißen (MORCZEK, coll. DEI, KARISCH), Lößnitz bei Radebeul (MÖBIUS, coll. MTD), Halbendorf/Spree (LEUTSCH), Baruth/Oberlausitz (LEUTSCH, WAUER), Ostritz/Oberlausitz (LEUTSCH, WAUER, GRAF), Oybin/Zittauer Geb. (LEUTSCH)

ST: Selketal/Harz (PATZAK, coll. DED)

TH: Jena (NICOLAUS, SCHADEWALD, coll. DEI), Bad Blankenburg (STEUER)

\section{Untersuchtes Material aus den übrigen Bundesländern:}

BW: Markgröningen (WÖRZ, coll. MTD)

BY: Arnstein/Oberfranken (NOWAK, coll. PRÖSE), Gambach/Unterfranken (PRÖSE), Kelheim (OSTHELDER, coll. ZSM, SEgERER), Regensburg (SÜLZ, coll. ZSM, SEGERER)

\section{Oegoconia deauratella (HERRICH-SCHÄFFER, 1854)}

Syst. Bearb. Schmett. Eur. 5: 135 (Lampros)

(= bacescui POPESCU-GORJ \& CAPUŞE, 1965)

Flugzeit: Ende Juni bis Ende August

Verbreitung: England, Niederlande, Dänemark, Finnland, Deutschland, Polen, Österreich, Italien (Sardinien), Ungarn, Rumänien, Bulgarien, Griechenland, Türkei, Russland, Kaukasus.

\section{Nachweise aus Ostdeutschland:}

MV: Bergen/Rügen (GAEDIKE, coll. DEI), Boltenhagen (ANGENSTEIN, coll. ZMHU), Dömitz/Elbe (SCHULZE, coll. DEI), Buchholz bei Rubow und Lübtheen (DEUTSCHMANN), Waren/Müritz (VAN LOH), NSG Serrahn bei Neustrelitz (FRIESE, coll. DEI), BB: Ferch bei Potsdam (LEUTSCH), Berlin-Friedrichshagen (FRIESE, coll. DEI), BerlinTreptow, Berlin-Marienfelde (STIESY, coll. DEI)

SN: Leipzig (E. MÜLLER, coll. NML), Meißen (MORCZEK, coll. DEI), Dresden-Blasewitz (coll. ZMHU), Demitz-Thumitz (BECK, KARISCH), Halbendorf/Spree, NSG Niederspree bei Dubitz (LEUTSCH), Großdubrau und Burg/Oberlausitz (GRAF)

ST: Staßfurt (SOFFNER, coll. DEI), Aschersleben (PATZAK, coll. DEI), Dessau (KELLNER), NSG Taubequelle Krs. Bitterfeld (SUTTER), Hohenlubast/Dübener Heide und Wittenberg (EICHLER, coll. ZSM)

TH: Gotha (LENTHE, coll. MNGO), Jena (RTTTER, coll. MNGE), Bad Blankenburg (STEUER)

\section{Untersuchtes Material aus den übrigen Bundesländern:}

NI: Leer und Rhede-Borsum (VAN LOH), Kohlerbissen und Wietzendorf (KAYSER)

MW: Erkelenz-Borschemich und Mönchengladbach-Rheydt (WIT"TLAND) 
Fundorte der Exemplare, von denen die Figuren stammen (alle Präparate vom Autor)

Fig. 1 Rheinland-Pfalz, Elmstein 19.7.1971 leg. RoESLER coll. ZSM, GU 6535; Fig. 2 Thüringen, Bad Blankenburg 28.8.1984 leg. und coll. STEUER, GU 6430; Fig. 3 BadenWürttemberg, Heidelberg-Kirchheim 20.7.1998 leg. RUTTEN, coll. SUTTER, GU 6510; Fig. 4 Thüringen, Jena 20.8.1976 leg. SCHADEWALD, coll. DEI, GU 6545; Fig. 5 BadenWürttemberg, Heidelberg-Kirchheim 4.7.1998 leg. und coll. RUTTEN, GU 6436; Fig. 6 Sachsen, Oberlausitz, NSG Niederspree, 27.6.1992, leg. und coll. LEUTSCH, GU 6405; Fig. 7 Thüringen, Umg. Jena 13.7.1960 coll. RITTER, coll. MNGE, GU 6571; Fig. 8 Rheinland-Pfalz, Dürkheim 12.7.1967 leg. RoESLER, coll. ZSM, GU 6564; Fig. 9 Rheinland-Pfalz, Elmstein 19.7.1971 leg. RoESLER coll. ZSM, GU 6535; Fig. 10 Baden-Württemberg, Heidelberg-Kirchheim 19.7.1998 leg. und coll. RUTTEN, GU 6441; Fig. 11 Bohemia, Rana bei Louny 22.8.1997 leg. und coll. SUTTER, GU 5356; Fig. 12 Thüringen, Bad Blankenburg 28.8.1984 leg. und coll. STEUER, GU 6430; Fig. 13 Sachsen, Oberlausitz, Schafberg bei Baruth 7.7.2000 leg. WAUER, coll. SUTTER, GU 6397; Fig. 14 Bulgaria, Pirin Mont., Bez. Sandanski, Liljanowo 27.6.-25.7.1985, leg. ErCHLER, coll. ZSM, GU 6552; Fig. 15 Baden-Württemberg, Heidelberg-Kirchheim 4.7.1998 leg. und coll. RUTTEN, GU 6436; Fig. 16 Niedersachsen, Emsland, Rhede-Borsum 27.6.1999 leg. und coll. vAN LOH, GU 6573; Fig. 17 Baden-Württemberg, Baden-Baden 14.8.1949 leg. LNACK, coll. ZSM, GU 6558; Fig. 18 Baden-Württemberg, Heidelberg-Kirchheim 22.7.1998 leg. und coll. RUTTEN, GU 6434; Fig. 19 Frankreich, 1880, coll. BANG-HAAS, coll. ZMHU, GU 6499; Fig. 20 Italia, Liguria, Noli 9.-20.6.1951, leg. KLIMESCH, coll. ZSM, GU 6417; Fig. 21 Sachsen, Oberlausitz, Steinberg bei Ostritz 11.8.2000 leg. und coll. WAUER, GU 6399; Fig. 22 Baden-Württemberg, Baden-Baden 14.8 .1949 leg. LINACK, coll. ZSM, GU 6558; Fig. 23 Rheinland-Pfalz, Neustadt/Pfalz 12.7.1973 leg. ROESLER, coll. ZSM, GU 6416; Fig. 24 Niedersachsen, Emsland, Rhede-Borsum 27.6.1999 leg. und coll. VAN LOH, GU 6573; Fig. 25 Sachsen-Anhalt, Staßfurt 16.8.1949 leg. SOFFnER, coll. DEI, GU 6456; Fig. 26 Sachsen, Oberlausitz, Demitz-Thumitz 28.7.1990 leg. und coll. BECK, GU 6167; Fig. 27 France, Les Landes, Contis 4.6.1999 leg. und coll. RUTTEN, GU 6433; Fig. 28 Spanien, Barcelona, coll. STAUDINGER, coll. ZMHU, GU 6505; Fig. 29 Kroatien, Istrien, Funtana 14.8.1971 leg. THOMAS, coll. MNVD, GU 6160; Fig. 30 Spanien, Barcelona, coll. STAUDINGER, coll. ZMHU, GU 6505; Fig. 31 Sachsen-Anhalt, Halle/Saale coll. O. MÜLLER, coll. ZMHU, GU 6500; Fig. 32 Frankreich, 1880, coll. BANG-HAAS, coll. ZMHU, GU 6499; Fig. 33 Italia, Liguria, Noli 9.-20.6.1951, leg. KuIMESCH, coll. ZSM, GU 6417; Fig. 34 Nederland, Prov. Gelderland, Wageningen-Hoog 4.7.1994, leg. und coll. KUCHLEIN, GU 6693; Fig. 35 Frankreich, 1880, coll. BANG-HAAS, coll. ZMHU, GU 6499; Fig. 36 Sachsen, Lößnitz bei Radebeul 2.8.1942, leg. MöBIUS, coll. MTD, GU 6467; Fig. 37 Sachsen, Oberlausitz, Steinberg bei Ostritz 11.8.2000 leg. und coll. WAUER, GU 6399; Fig. 38 Thüringen, Bad Blankenburg 28.8.1984 leg. und coll. STEUER, GU 6431; Fig. 39 Bayern, Regensburg 12.8.1918 leg. SÄLZL, coll. ZSM, GU 6563; Fig. 40 Sachsen, Lößnitz bei Radebeul 2.8.1942, leg. MÖBIUS, coll. MTD, GU 6467. 


\section{Literaturverzeichnis}

BIESENBAUM, W. 2002: Revision der bisher in unserem Arbeitsgebiet festgestellten Oegoconia-Arten (Lep., Autostichidae). - Melanargia, Leverkusen 14 (1): 4-9.

GAEDIKE, R. \& HEINICKE, W. (Htsg.) 1999: Verzeichnis det Schmetterlinge Deutschlands. - Entomologische Nachrichten und Berichte, Dresden Beiheft 5: 1-126 (Entomofauna Germanica 3).

HuEmer, P. 1998: Neue Erkenntnisse zur Identität und Verbreitung europäischer Oegoconia-Arten (Lepidoptera, Autostichidae). - Mitteilungen der Münchner Entomologischen Gesellschaft, München 88: 99-117.

SUTTER, R. 2001: Zur Identität von Oegoconia ariadne GOZMÁNY,1988 (Lep., Symmocidae). - Entomologische Nachrichten und Berichte, Dresden 45 (2): 111-114.

SUTTER, R. \& LIŠKA, J. 2002: Oegoconia uralskella corsa ssp. n. (Insecta: Lepidoptera: Autostichidae). Entomologische Abhandlungen, Dresden 60: 73-78.

\section{Anschrift des Verfassers:}

REINHARD SUTTER,

Fläminger Ufer $4 \mathrm{a}$

D - 06749 Bitterfeld 


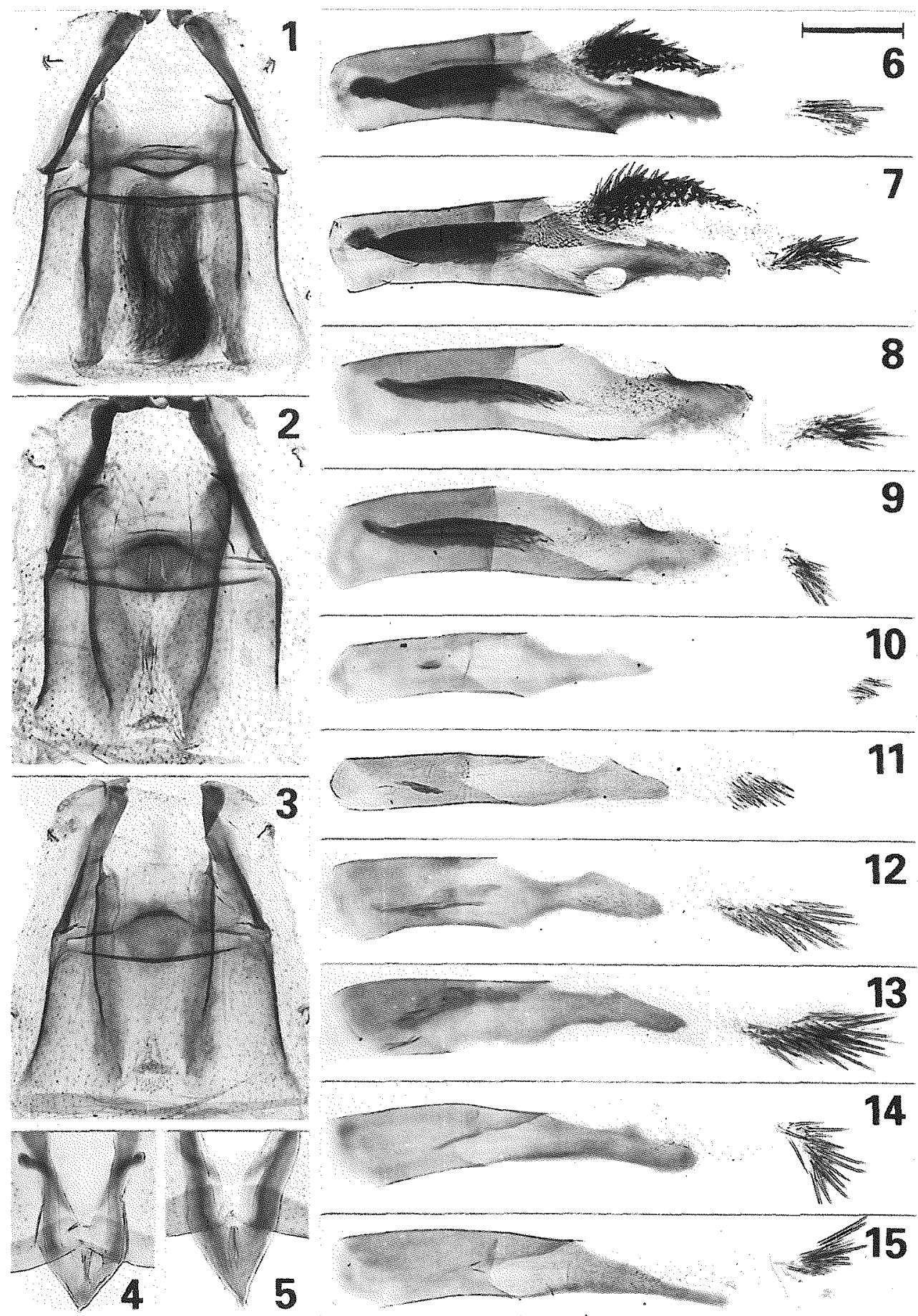

Fig. 1-15: Oegoconia sp., o Sternit I-II, Saccus, Aedoeagus (Maßstab bei Fig. 4-15: 0,2 mm): Fig. 1, 8, 9 quadripuncta; Fig. 2, 4, 12, 13 uralskella; Fig. 3, 5, 14, 15 novimundi; Fig. 6, 7 deauratella; Fig. 10, 11 caradjai. 

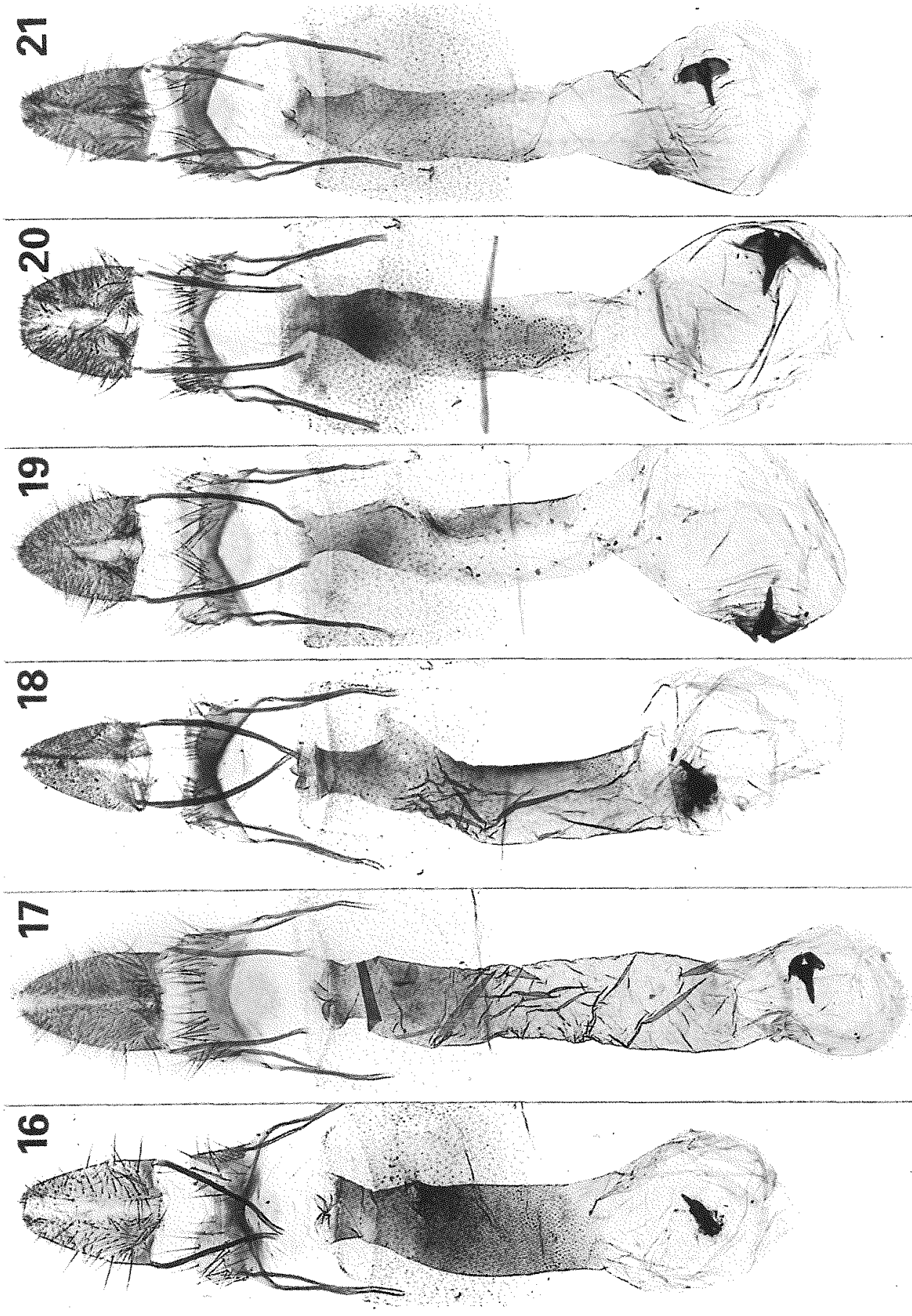

Fig. 16-21: Oegoconia sp., Genital ㅇ: Fig. 16 deauratella; Fig. 17 quadripuncta; Fig. 18 novimundi; Fig. 19, 20 caradjai; Fig. 21 uralskella. 


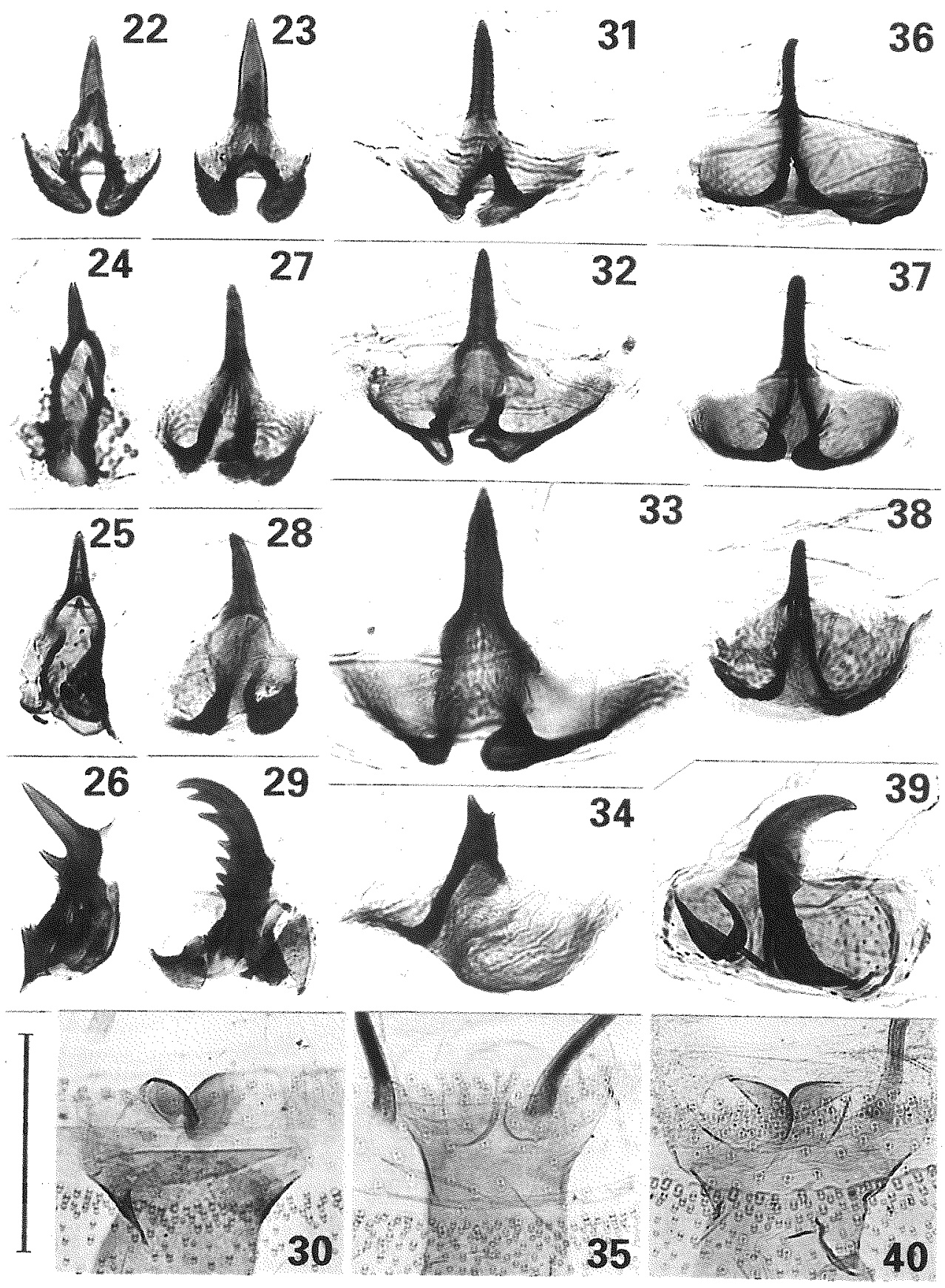

Fig. 22-40: Oegoconia sp., o Signum, Ostium (Maßstab: 0,2 mm): Fig. 22-23 quadripuncta; Fig. 24-26 deauratella; Fig. 27-30 novimundi; Fig. 31-35 caradjai; Fig. 36-40 uralskella. 